\title{
Representação Social do Sexo nos Jovens Adultos Portugueses
}

\author{
Social Representation of Sex in Portuguese Young Adults
}

\author{
Alexandra Gomes* \& Cristina Nunes \\ Research Centre for Spatial and Organizational Dynamics, Universidade do Algarve, Faro, Portugal
}

\begin{abstract}
Resumo
Foi nosso objetivo medir a representação social do sexo através das evocações livres de 1138 jovens adultos portugueses, com idades entre os 18 e os 25 anos, explorando uma abordagem explicativa da possível influência desta representação social nos comportamentos sexuais de risco observados nesta faixa etária. As evocações foram recolhidas através de um questionário online, divulgado pelas universidades e escolas profissionais portuguesas. Foi realizada uma análise prototípica de representações sociais. Os resultados sugerem uma representação social do sexo romantizada, com um núcleo central baseado na ideia de amor e prazer, em que aspetos relacionados com o risco, como preservativo ou infeções sexualmente transmissíveis, pertencem apenas ao sistema periférico, considerando-se que aí são integrados como uma forma de preservar o núcleo central, mas sem interferir na forma como os participantes vivem as suas experiências sexuais. A associação entre a ideia de amor e preservativo parece estar mais associada à heurística de quem "ama protege" o que facilita a não utilização do preservativo, em vez de a aumentar. A representação social do sexo, centrada no amor e no prazer, poderá enquadrar as questões cognitivas, afetivas e comportamentais presentes na explicação e predição da utilização do preservativo, complementando os modelos sociocognitivos já conhecidos. Palavras-chave: Representação social, comportamentos sexuais de risco, uso do preservativo, análise prototípica.
\end{abstract}

\begin{abstract}
The objective of this study was to measure the social representation of sex through free evocations of 1,138 young Portuguese adults, aged between 18 and 25 years, with the goal of exploring an explicative approach of a possible influence of this social representation in risky sexual behaviors observed in this age group. Evocations were collected through an online questionnaire made available by Portuguese universities and professional schools. Data were analyzed according to Vergès' prototypical analysis for social representations. The results suggest a romanticized social representation of sex, based on love and pleasure ideas, in which risky aspects such as use of condoms or sexually transmitted infections belong to the peripheral system. These aspects are integrated in order to preserve the central nucleus without interfering in the way participants live their sexual experiences. In addition, the association between love and condom use seems to be associated to the concept of "who loves, protects" which reduces condom use, instead of increasing it. Therefore, social representation of sex, focused on love and pleasure, might include cognitive, affective and behavioral aspects present in the well-known socio-cognitive models used for explaining and predicting condom use behavior.

Keywords: Social representation, risky sexual behavior, condom use, prototypical analysis.
\end{abstract}

A representação social constrói-se na interação com o meio e permite a orientação dos comportamentos (Moscovici, 1961), mesmo que de forma inconsciente como observado por Jodelet (1989). Contudo, os estudos que procuraram relacionar as representações sociais e os comportamentos pressupõem que os indivíduos controlam as representações sociais e selecionam as respostas da forma que lhes parece mais adequada (cf. Abric, 1987).

Para Vala (2004) as representações incluem os modos desejáveis de ação, proporcionam a construção do

\footnotetext{
" Endereço para correspondência: Departamento de Psicologia e Ciências da Educação, Universidade do Algarve, Faculdade de Ciências Humanas e Sociais, Campus de Gambelas, Edf. 1, Gambelas, Faro, Portugal 8005-139. E-mail: asgomes@ualg.pt

Este artigo foi parcialmente financiado pela Fundação para a Ciência e Tecnologia (FCT, Portugal).
}

significado do estímulo em contexto e permitem ainda justificar os comportamentos individuais. Abric (2003) refere que as mudanças introduzidas no núcleo central de dada representação social provocam alterações ao nível das atitudes, enquanto as alterações nas atitudes apenas produzem mudanças no sistema periférico. Para Flament (2001) são os elementos periféricos que fazem a ponte entre os constructos mais abstratos do núcleo central e os contextos de utilização da representação, atuando como esquemas ou scripts de ações relativas ao objeto da representação social.

Apesar de não existir um modelo geral que permita compreender as suas relações (Wachelke \& Camargo, 2007), vários estudos dos comportamentos sexuais de risco, o uso do preservativo e o HIV têm procurado utilizar esta ligação entre as representações sociais e o comportamento, como forma de explicar o hiato existente entre 
intenção e comportamento (por exemplo, Braun, 2013; Giacomozzi \& Camargo, 2011).

Como exemplos, Morin e Vergès (1992) procuraram definir a representação social da Aids nos adolescentes do ensino secundário. A Aids apresentava uma dupla configuração central, com os conceitos de morte e doença a apresentarem a maior saliência para os jovens. Contudo, a representação apresentava-se complexa. As noções de amor e confiança estavam em conflito com a noção de sexualidade, que por sua vez estava relacionada com noções de risco. Do ponto de vista preventivo, as noções de amor e confiança estavam ligadas a discussão e informação.

Goodwin e colaboradores (2004) num estudo sobre a representação do HIV/Aids em 5 países da Europa central e de leste encontraram duas grandes dimensões, categorizadas como "sexo" e "doença mortal", com forte congruência. Contudo, os resultados sugerem uma maior inconsistência ao nível da dimensão "sexo", em parte influenciada pelo contexto cultural de cada país. Esta dimensão fazia referência à existência de episódios de sexo casual, preservativos, prostituição e atividade sexual em geral, que refletia a componente sexual da epidemia. Por sua vez, a dimensão "doença mortal" enfatizava o aspeto do medo relacionado com a doença Aids.

Camargo, Barbará e Bertoldo (2007), num estudo sobre a representação social da Aids com 262 jovens brasileiros verificaram que os participantes centravam a sua representação nos elementos de doença, morte, medo, sofrimento, preconceito, mas também prevenção e responsabilidade. As representações variavam consoante o grau de informação dos participantes sobre o HIV, existindo uma maior preocupação relacionada com aspetos biomédicos nos indivíduos mais informados. Os autores sugerem que o esforço informacional sobre o HIV/Aids é favorável à transformação da representação social da Aids, aumentando a consciência do risco e promovendo os comportamentos sexuais seguros.

Camargo e Bousfield (2009) analisaram a relação entre a representação social da Aids e os motivos enumerados pelos jovens para a não utilização do preservativo. Os resultados sugerem a formação de três dimensões de resposta para o facto de não utilizarem preservativo: (a) a excitação no momento da relação sexual, não permite um raciocínio claro, conduzindo a situações desprotegidas, (b) a existência de confiança na relação e no parceiro que conduz à ilusão de que não correm riscos devido a uma relação monógama e, (c) a utilização de outro método contracetivo, evidenciando uma maior preocupação com as gravidezes indesejadas do que com as infeções sexualmente transmissíveis.

Bertoldo e Barbará (2006) estudaram a representação social do namoro, na visão dos jovens brasileiros. Os resultados sugerem que para os jovens adultos brasileiros o namoro é descrito em termos de uma amizade, cumplicidade, confiança e amor. Apesar de o estudo não se centrar diretamente nos comportamentos de risco, como a incon- sistência do uso do preservativo, permite compreender que há uma elevada valorização de aspetos de intimidade nas relações de namoro dos jovens adultos brasileiros. Já o estudo desenvolvido por Giacomozzi e Camargo (2004) com uma amostra de mulheres brasileiras com parceiro estável, mostra que as participantes relacionam a segurança e confiança no parceiro como uma forma de proteção face às doenças sexualmente transmissíveis, como o HIV/ Aids. Oltramari e Camargo (2004) observaram ainda que inclusive em mulheres profissionais do sexo, não existia a preocupação de utilização de medidas de proteção sexual com os seus parceiros fixos, comportamento associado à mesma ideia de confiança e amor, o que as coloca num nível de risco mais elevado em relação a infeções sexualmente transmissíveis.

Bromnick e Swinburn (2003) num estudo sobre as representações das experiências sexuais de uma amostra de adolescentes analisaram o conteúdo de entradas de diário sobre possíveis experiências sexuais desprotegidas. Os resultados sugerem que os adolescentes não consideram a experiência de sexo sem preservativo como algo mau ou reprovável. Apenas uma minoria escreveu nos diários aspetos relacionados com a saúde ou sobre preocupações que lhe estivessem associadas. Os aspetos românticos ou relacionados com a vivência sexual (satisfação, comprometimento) foram considerados mais importantes do que os da saúde. Estes estudos salientam a relevância do estudo das representações sociais para compreender os comportamentos sexuais de risco, e em particular, o uso do preservativo.

Mais recentemente Gomes e Nunes (2011a) procuraram medir a representação social do sexo veiculada através do discurso social, extraído de uma amostra trimestral de jornais e revistas portuguesas. Os resultados mostram dois tipos de discursos, um vocacionado para aspetos criminalizados do sexo, e outro mais vocacionado para aspetos funcionais e relacionais, como fertilidade, prazer, e da relação de casal. É ainda de notar que o corpus recolhido não fazia referências significativas a aspetos relacionados com o uso do preservativo ou doenças sexualmente transmissíveis, mostrando um lado romantizado e vocacionado para o amor e o prazer, mas não para o risco. Braun (2013) através da análise dos discursos através da análise de conteúdo de grupos focais, relativos ao preservativo, desenvolve a metáfora do preservativo enquanto aniquilador do prazer, mostrando que há um discurso negativo e oposto ao observado no estudo anterior, que contextualiza a relação sexual como sendo algo bom e positivo.

Como tal, este estudo tem como objetivo definir a estrutura da representação social do sexo, dado que as representações sociais têm um papel na determinação do comportamento dos indivíduos. A noção de que o sexo é um dos conceitos de maior associação ao HIV/Aids, e sendo o contexto em que se utiliza o preservativo, procurou-se compreender de que forma se estrutura a representação social do sexo, numa amostra de jovens adultos que, de 
acordo com o relatório sobre o estado da infeção por HIV/ Aids (Instituto Nacional de Saúde [INS], 2013), são o grupo que continua a registar a maior incidência de novas infeções em Portugal.

\section{Método}

\section{Participantes}

A amostra recolhida foi recrutada de forma não probabilística entre os estudantes do ensino superior e de escolas profissionais portuguesas. Os participantes deveriam ter entre 18 e 25 anos e nacionalidade portuguesa, o que deu elegibilidade a 1138 respostas das 1682 obtidas. A média das idades foi de 20,93 anos ( $D P=2,11), 33,00 \%$ são homens e $67,00 \%$ são mulheres. Cerca de $14,50 \%$ da amostra era trabalhador-estudante, sendo os restantes estudantes, provenientes em $88,52 \%$ de sistemas universitários portugueses e $11,48 \%$ de centros e escolas profissionais. Um total de $98,50 \%$ dos participantes era solteiro.

\section{Instrumento}

Foi construído um questionário que abordava as questões demográficas, a história sexual (parceiros, iniciação da atividade sexual, tipos de relações sexuais) e hábitos de uso do preservativo (utilização nas relações sexuais, frequência da utilização, situações de inconsistência e intenção de uso). A representação social foi apurada através de uma tarefa de evocação livre. Era pedido aos participantes para referirem que palavras ou expressões lhes eram sugeridas pela palavra Sexo. Para o efeito foram fornecidos 15 espaços em branco, que deveriam preencher, considerando que a ordem das palavras deveria corresponder à ordem de importância relativa para o próprio. Era clarificado que os participantes poderiam preencher os espaços que considerassem necessários.

\section{Procedimento}

Inicialmente o estudo foi proposto ao Conselho Científico da Faculdade de Ciências Humanas e Sociais, da universidade do Algarve, que o aprovou. Em seguida, foi construída uma página da internet, com o questionário, o que permitiu a sua divulgação por todos os possíveis participantes. Para abranger o máximo de jovens adultos, foram contactadas todas as universidades públicas portuguesas, através de um pedido formal, endereçado aos respetivos reitores. Foram, também, contactadas escolas profissionais cujos cursos fossem direcionados para jovens adultos.

Após a aprovação dos respetivos órgãos dirigentes e científicos, o pedido de participação no estudo com o respetivo endereço online do questionário foi divulgado através do correio eletrónico institucional. A folha de rosto do questionário informava sobre o objetivo do trabalho, referindo-se a um estudo sobre o comportamento sexual dos jovens adultos, e continha uma premissa de consentimento informado. Os participantes que desejassem participar deveriam selecionar uma opção (um botão) que dava prosseguimento ao questionário. Caso não pretendessem participar, era fornecida uma opção que permitia fechar automaticamente a página. Eram igualmente informados que podiam desistir em qualquer altura, sem qualquer consequência associada. No fim do questionário, era agradecida a participação e fornecido o endereço eletrónico do investigador responsável, para quaisquer informações, críticas ou sugestões que pretendessem realizar ou fornecer.

Os dados recolhidos foram analisados com recurso ao SPSS v. 17, bem como ao programa EVOC2000 (Scano, Junique, \& Vergès, 2002). A técnica de Vergès (1992), denominada de análise prototípica, cruza dois critérios para definir quais as palavras que poderão fazer parte do núcleo central de uma representação social: (a) ordem média de evocação e (b) frequência em que a palavra/ categoria ocorre. De acordo com a análise, as palavras com frequência elevada e evocadas nas primeiras posições serão provavelmente os elementos que constituem o núcleo central.

No presente caso, a análise das respostas foi realizada recorrendo às palavras brutas, tendo existido um agrupamento exclusivo em casos de semelhança radial, que não contemplasse alterações de significado essencial (por exemplo: abraços, abraço e abraçar). Nesta perspetiva reduziram-se as formas verbais à forma mais simples, os plurais a singular e uniformizou-se o género para a forma masculina. As expressões, das quais são exemplos fazer amor ou atividade sexual, foram contempladas como sendo uma forma única.

Para determinar os valores de frequência mínima e intermédia, bem como a ordem média de evocação correta a adotar, foram observados alguns estudos sobre representações sociais (consultar Tabela 1). A frequência mínima escolhida corresponde a, pelo menos, $5 \%$ da amostra, o que equivale às palavras evocadas pelo menos 56,95 vezes. A frequência intermédia foi escolhida através da observação qualitativa. Verificou-se que a $2^{\mathrm{a}}$ e $3^{\mathrm{a}}$ palavras mais evocadas têm uma frequência na ordem dos $583 \mathrm{e}$ 221 (evocadas por $51,10 \%$ e $19,40 \%$ da amostra, respetivamente). Considerou-se que esta diferença era suficiente para constituir um corte qualitativo, determinando-se que a frequência intermédia se situava nos 583. A ordem média das evocações foi calculada através da média aritmética da ordem de evocação de palavras. O valor obtido foi de $4,33(\approx 4)$.

\section{Resultados}

Caracterização da História Sexual e do Uso do Preservativo

A maioria dos participantes reportou já ter iniciado a sua vida sexual $(72,90 \%)$ tendo como prática sexual mais comuns as relações vaginais e orais $(26,00 \%)$. A média da idade primeira relação sexual situava-se nos 17,51 $(D P=3,49)$ anos e a do parceiro nos $19,44(D P=5,64)$. A média do número total de parceiros foi de $3,36(D P=5,72)$ e nos últimos 3 meses foi de $1,126(D P=3,52)$. Aquando da aplicação do questionário $54,80 \%$ da amostra referia ter parceiro. 
Psicologia: Reflexão e Crítica, 28(1), 177-185.

Tabela 1

Critérios de Análise das Evocações

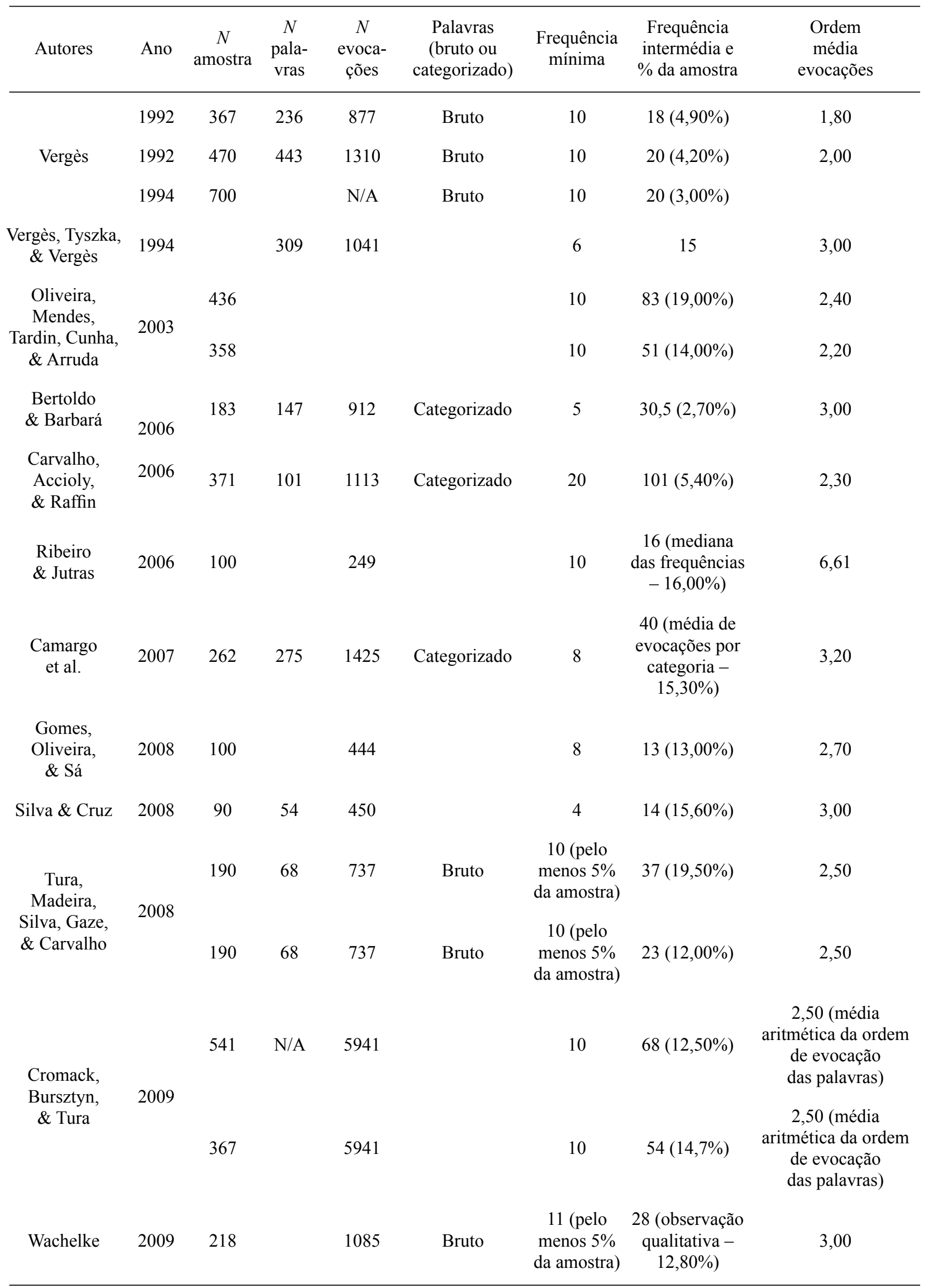


Gomes, A. \& Nunes, C. (2015). Representação Social do Sexo nos Jovens Adultos Portugueses.

Quanto ao uso do preservativo, a média de utilização foi de 4,86 ( $D P=2,21)$ com uma mediana de 6 (escala de 1 nunca a 7 sempre). Cerca de $57,66 \%$ dos participantes, que já iniciaram a sua vida sexual, afirmaram ter utilizado preservativo na última relação sexual. Em média, nos últimos 6 meses, os participantes não utilizaram o preservativo $10,29(D P=22,08)$ vezes, e no último mês cerca de 3,31 $(D P=11,10)$. A intenção futura de uso do preservativo teve uma média de 5,29 ( $D P=2,24$; escala de 1 nunca a 7 sempre).
Análise Prototípica da Amostra Geral

Foi observado um total de 7183 evocações referente a 1396 palavras diferentes. A aplicação da frequência mínima reduziu o número de palavras a 21 , evocadas 2923 vezes. A ordem média de evocação foi de 4,30, o que permite apurar que os participantes realizaram em média entre quatro e cinco evocações na tarefa de associação livre.

A frequência intermédia considerada permitiu dividir as palavras evocadas por quatro quadrantes (ver Tabela 2).

Tabela 2

Estrutura da Representação Social do Sexo

\begin{tabular}{|c|c|c|c|c|c|c|}
\hline & \multicolumn{3}{|c|}{ Ordem Média de Evocação $(\mathrm{OME})<4,3$} & \multicolumn{3}{|c|}{ Ordem Média de Evocação (OME)>4,3 } \\
\hline & Palavra & $f$ & OME & Elemento & $f$ & OME \\
\hline \multirow[t]{2}{*}{$f \geq 583$} & Amor & 631 & 2,45 & & & \\
\hline & Prazer & 583 & 3,21 & & & \\
\hline \multirow[t]{10}{*}{$f \leq 583$} & Bom & 91 & 2,78 & Cama & 56 & 5,39 \\
\hline & Carinho & 153 & 4,19 & Corpo & 65 & 4,75 \\
\hline & Diversão & 60 & 4,20 & Cumplicidade & 56 & 4,62 \\
\hline & Homem & 75 & 3,25 & Desejo & 134 & 4,59 \\
\hline & Intimidade & 118 & 3,85 & $\begin{array}{l}\text { Doenças sexualmente } \\
\text { transmissíveis }\end{array}$ & 66 & 7,41 \\
\hline & Mulher & 100 & 3,41 & Loucura & 74 & 5,01 \\
\hline & Namorado & 72 & 3,81 & Orgasmo & 74 & 5,81 \\
\hline & Paixão & 221 & 3,81 & Partilha & 70 & 4,81 \\
\hline & Relação & 95 & 3,10 & Preservativo & 71 & 5,68 \\
\hline & & & & União & 67 & 4,85 \\
\hline
\end{tabular}

O primeiro quadrante, com uma alta frequência e alta ordem média de evocação, contém apenas duas palavras, Amor e Prazer. Estas palavras poderão constituir os elementos mais centrais da representação. Amor é a palavra com maior frequência e que aparece nas primeiras posições, mais frequentemente. Não se verificou a existência de palavras com uma frequência tão elevada e uma ordem média de evocação superior a 4,3, o que sustenta a centralidade dos vocábulos anteriores.

Com frequência inferior a 583, mas com uma ordem média de evocação superior, encontram-se diversas palavras, em parte relacionadas com os aspetos relacionais do sexo (homem, mulher, relação, intimidade, namorado, carinho) ou com aspetos relacionados com o prazer ou consequências positivas do sexo (bom, diversão, paixão).
As palavras com baixa frequência e com baixa ordem de frequência poderão ser as que constituem o elemento mais periférico da representação. Continuam-se a registar aspetos mais associados à relação (cumplicidade, partilha, união), aspetos relacionados com prazer mas de um ponto de vista mais físico ou das práticas sexuais (corpo, desejo, loucura, orgasmo, cama) e ainda aspetos que se prendem com o risco/prevenção (preservativo, doenças sexualmente transmissiveis).

\section{Análise de Coocorrências}

Com recurso do programa SIMI 2000 (Junique, Barbry, Scano, Zeliger, \& Vergès, 2002) foram verificadas as coocorrências das palavras mais evocadas pelos participantes do estudo. Por se considerar que o agrupamento em categorias iria enviesar a análise conduzida, optou-se por realizar a análise com as 21 palavras brutas. 


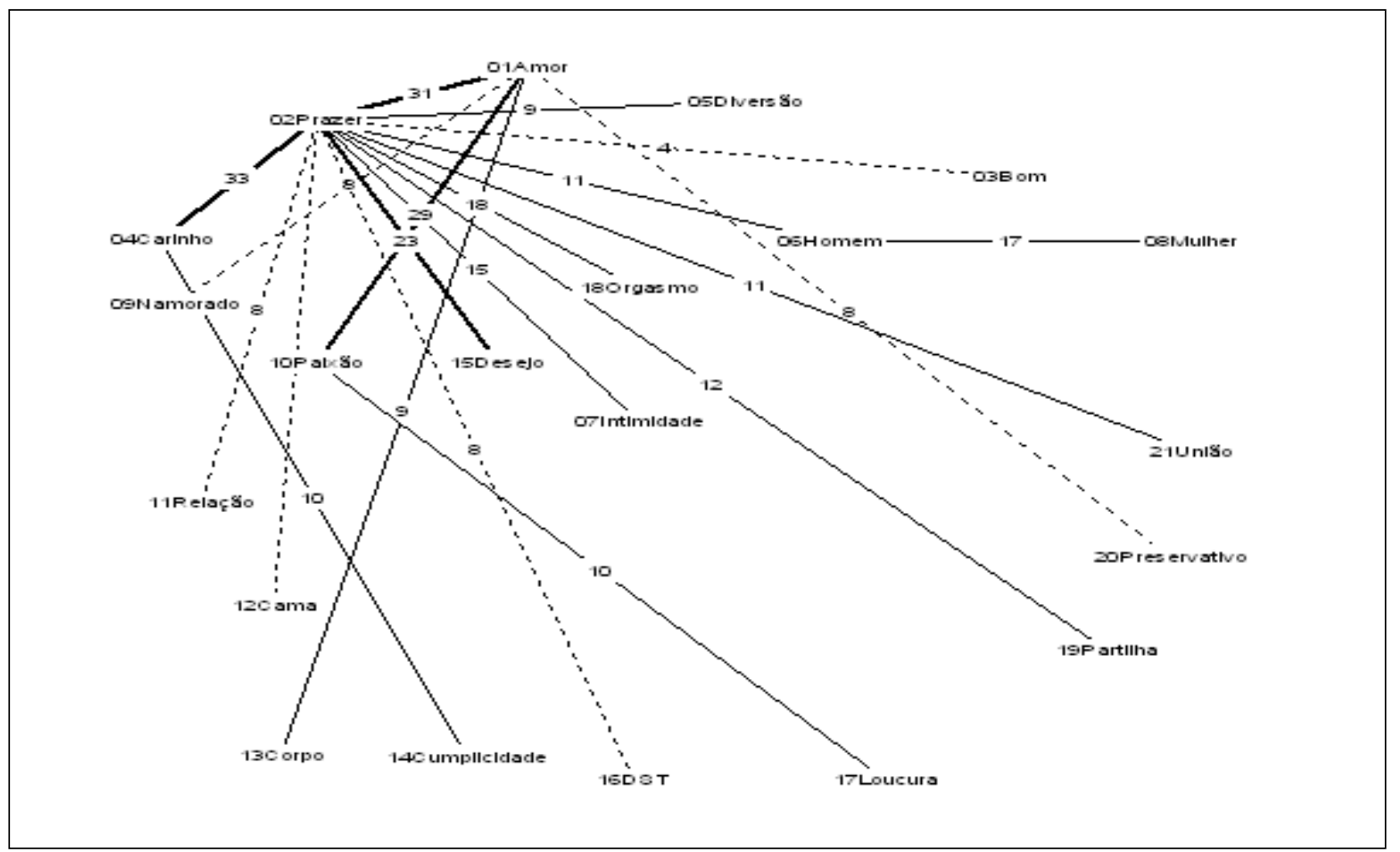

Figura 1. Coocorrências das palavras evocadas.

Através da análise das frequências absolutas das coocorrências das palavras evocadas (ver Figura 1) verificou-se que ambas as palavras apontadas como mais centrais pelos participantes estabeleciam ligações com as palavras mais periféricas. A palavra Prazer foi a que estabelecia mais ligações, primordialmente associada ao Carinho e, secundariamente, ao Amor. O Prazer estava igualmente ligado a Desejo, Intimidade, Partilha, Orgasmo, União, Homem - que estabelecia uma ligação terciária a Mulher -, Diversão, Relação, Cama e, finalmente Bom.

$\mathrm{O}$ Amor estabelecia menos relações, surgindo ligado a Paixão, que se encontrava por sua vez, associada a Loucura. É igualmente o Amor que estabelecia relações com o Preservativo e Namorado. Mas foi Prazer que apareceu associado a Doenças sexualmente transmissiveis.

\section{Discussão}

Foram evocadas 21 palavras por, pelo menos, 5\% da amostra, em que sobressaem dois conceitos com elevada ordem de frequência e evocação: Amor e Prazer. É observável que a natureza das palavras é semelhante, em significado, às palavras presentes no núcleo central. No entanto, a utilização das palavras brutas, em vez das palavras agrupadas em categorias, foi considerada mais adequada para evitar perdas de informação (Vergès, 1992). O sistema periférico inclui palavras muito pouco frequentes nas evocações da amostra total, mas é de notar que faz referência tanto ao preservativo como às doenças sexualmente transmissíveis.
Em relação ao comportamento de utilização do preservativo, as médias encontradas são semelhantes às de estudos desenvolvidos com amostras portuguesas (Gomes \& Nunes, 2011b; Muñoz-Silva, Sánchez-García, Martins, \& Nunes, 2009, Reis, Ramiro, Matos, \& Diniz, 2013). É ainda de notar que a média de utilização do preservativo na amostra revela-se inconsistente, apesar de uma elevada intenção de uso. Estudos anteriores já tinham observado uma romantização da experiência sexual que se estendida inclusivamente às experiências sexuais desprotegidas (Bromnick \& Swinburn, 2003; Giacomozzi \& Camargo, 2004). Apesar de não ser possível garantir que ambas as amostras partilham a mesma representação social, o núcleo central focado no Amor e Prazer parece ser uma explicação plausível para esta atribuição.

Igualmente, no estudo de Camargo e Bousfield (2009) os aspetos românticos do sexo justificavam a não utilização do preservativo. Confiar e amar o parceiro surge como um enviesamento percetivo do risco, o que o aumenta perante as doenças sexualmente transmissíveis.

Os estudos em Portugal sobre o uso do preservativo mostram uma diminuição deste em detrimento da pílula, pela perda da sensação e prazer que lhe está associada (Ministério da Saúde, 2005). Apesar dos indivíduos reconhecerem o risco do HIV/Aids, aumentado pelo não uso do preservativo, a perceção individual do risco diminui quando se consideram as experiências individuais (Amaro, Frazão, Pereira, \& Teles, 2004). Verifica-se, igualmente que a utilização do preservativo diminui claramente perante a existência de um parceiro considerado estável 
(Muñoz-Silva et al., 2009). Considerando o núcleo central observado na representação social do sexo, poder-se-á supor que as noções de amor e prazer poderão justificar os níveis de utilização do preservativo.

Os aspetos formais do risco e da proteção surgem integrados no sistema periférico da representação social mas não ao ponto de ameaçarem o conteúdo central, o Prazer e o Amor. Desta forma, é plausível compreender-se, porque apesar do conhecimento da existência e transmissão de infeções sexuais (por exemplo, Reis et al., 2013), o risco pessoal é geralmente percecionado como sendo inferior ao real, facto presente tanto em amostras portuguesas (Amaro et al., 2004), como em amostras não portuguesas (Adefuye, Abiona, Balogun, \& Lukobo-Durrell, 2009; Kayiki $\&$ Forste, 2012). Deve-se, igualmente, considerar que a representação social integra informação no seu sistema periférico, sem no entanto modificar os valores e crenças centrais. Tal poderá permitir a coexistência de informação contrária, mas que poderá ativar-se ou desativar-se mediante determinadas condições (Flament, 2001; Wachelke \& Camargo, 2007).

Estas associações observam-se, ainda através das relações de coocorrência entre as palavras pertencentes ao núcleo central, e as restantes palavras que integram a representação, incluindo as que estão definidas como sistema periférico.

A palavra Amor coocorre com mais frequência com as palavras Prazer, Paixão, Corpo, Namorado, Preservativo, sendo que por sua vez, a palavra Paixão coocorre com Loucura. A perspetiva do amor estar associado a aspetos como paixão e loucura, bem como namorado e preservativo, parece contextualizar a ideia de que o amor é algo apenas partilhado com relações mais estáveis, apesar de continuarem a figurar os aspetos arrebatadores do prazer, da paixão e da loucura. $\mathrm{O}$ enquadramento do preservativo neste cenário parece ser uma forma de proteção e segurança, associadas ao amor.

Os dados das investigações com amostras portuguesas e o uso do preservativo evidenciam que os parceiros mais velhos e com parceiros fixos (namorados) são os que utilizam em menor proporção o preservativo (Gomes \& Nunes, 2011b; Muñoz-Silva et al., 2009; Reis, Ramiro, \& Matos, 2009), sendo que o mesmo se passa em amostras internacionais (Bogart et al., 2005; Matson, Adler, Milsstein, Tschann, \& Ellen, 2011). Desta forma, parece que as associações amor-namorado, e amor-preservativo, poderão indiciar mais uma forma de justificar a não utilização do preservativo do que constituir uma possibilidade do seu uso. Este facto poderá estar a contextualizar heurísticas cognitivas que evidenciam que "quem ama, está protegido" contra doenças sexualmente transmissíveis, o que está associado a uma diminuição da utilização do preservativo (Ayala, Rivas, \& Bingham, 2005; Camargo \& Bousfield, 2009).

Por sua vez, o Prazer estabelece relações com todos os restantes vocábulos, mostrando uma perspetiva organizadora da representação social do sexo. A conceptualização do prazer revela noções de relacionamento, de diversão, de intimidade e cumplicidade, mas coocorre igualmente com as doenças sexualmente transmissíveis. Provavelmente, os indivíduos que se centram mais no atributo do Prazer estarão mais conscientes dos riscos adotados numa perspetiva da vivência da sexualidade. $O$ estudo realizado por Gebhardt, Kuyper e Greunsven (2003) comparou a utilização do preservativo entre participantes com parceiro estável e participantes com parceiros casuais. Observaram que a utilização do preservativo era mais comum no segundo grupo, sendo que estes utilizavam mais o preservativo quanto mais favorável fosse a atitude e quanto maior a autoeficácia de uso e necessidade de intimidade nas relações. A associação entre o prazer e a intimidade pode, portanto, contextualizar a necessidade da mesma para que os parceiros comuniquem e consigam utilizar o preservativo. Mais recente, um estudo qualitativo sobre a representação das doenças sexualmente transmissíveis e a relação com uso o preservativo nas relações sexuais por homens heterossexuais refere a diminuição do preservativo em situações de maior intimidade e afetividade com as parceiras, relatando o desconforto e falta de sensibilidade com que o caracterizam (Pires \& Balieiro, 2012).

Continua-se, portanto, a observar que uma das maiores barreiras ao preservativo é a falta de prazer e a interferência com a excitação sexual (Braun, 2013; Castro-Vazquez \& Kishi, 2007; Kaneko, 2007; Ministério da Saúde, 2005). Manuel (2005) identificou que a interpretação pessoal de prazer dos participantes, juntamente com a falta de educação sexual e de informação sobre HIV, contribuíam para a não utilização do preservativo. Portanto, a representação social do sexo, centrada no amor e no prazer, poderá enquadrar as questões cognitivas, afetivas e comportamentais presentes na explicação e predição da utilização do preservativo.

\section{Considerações Finais}

Este estudo teve como objetivo definir a estrutura da representação social do sexo, contribuindo, desta forma, para a compreensão de comportamentos de risco no campo da sexualidade, especificamente na utilização do preservativo entre o grupo mais vulnerável à infeção por HIV. Os critérios considerados para a identificação de uma representação social (cf. Vala, 2004; Wachelke \& Camargo, 2007), permitem-nos afirmar que estamos perante uma representação generalizada, coletivamente gerada, e que parece suportar guias de ação e permitir a interpretação de comportamentos dos indivíduos. Não obstante, algumas considerações devem ser tecidas ao estudo. A amostra recolhida refere-se a uma dimensão específica dos jovens adultos portugueses, isto é, aqueles que ainda estão inseridos em sistemas de ensino. Seria relevante que, de futuro, existisse um método de amostragem mais equilibrado que nos permitisse recolher respostas de indivíduos com outras experiências de vida, e suportando a validade externa do estudo. De forma semelhante observa-se um desequilíbrio de sexos na amostra. As mulheres surgem numa clara maioria, que deriva do facto de ser uma amostra mais escolarizada. Os últimos censos realizados em Portugal 
(Instituto Nacional de Estatística, 2011) mostram que são as mulheres que maioritariamente prosseguem estudos, sendo portanto mais provável encontrar um maior número de respondentes deste sexo. Contudo este aspeto poderá enviesar os resultados do estudo, pelo que os resultados devem ser analisados com a devida cautela.

São também necessários mais estudos que permitam explorar melhor a relação entre a representação social e o comportamento. O nosso objetivo não pretendia criar um modelo, contudo seria relevante compreender melhor o impacto da representação social na explicação do comportamento, como forma organizadora dos comportamentos individuais. Notamos também, que a representação social do sexo deve ser enquadrada numa perspetiva relacional, já que os indivíduos que fazem parte da dinâmica da relação sexual, poderão partilhar a mesma representação. Este denominador comum entre as partes poderá ajudar a explicar porque há um hiato entre intenção e comportamento, visível nos estudos que se baseiam nos modelos intenção-ação.

Estes estudos futuros permitirão validar os avanços para a população portuguesa, onde as taxas de HIV continuam a aumentar entre os jovens adultos, acompanhado de uma diminuição da utilização do preservativo (INS, 2013), e onde continuam a ser insuficientes as investigações que permitem compreender este comportamento. Numa perspetiva mais geral, a caracterização da representação social do sexo em outras culturas, e as respetivas comparações, poderiam contextualizar as diferenças encontradas ao nível o uso do preservativo, apesar de os países ocidentalizados dos Estados Unidos da América e Europa Ocidental e Central partilharem os mesmos indicadores referentes à utilização do preservativo e infeção de HIV/Aids (Joint United Nations Program on HIV/AIDS [UNAIDS], 2012). Futuramente propomo-nos observar diferenças ao nível da representação social do sexo com base nas experiências individuais e hábitos de utilização do preservativo.

\section{Referências}

Abric, J. C. (1987). Coopération, compétition et représentation socials. Cousset, Suisse: DelVal.

Abric, J. C. (2003). Abordagem estrutural das representações sociais: Desenvolvimentos recentes. In P. H. F. Campos \& M. C. da S. Loureiro (Eds.), Representações sociais e práticas educativas (pp. 37-57). Goiânia, GO: Editora da Pontifícia Universidade Católica de Goiás.

Adefuye, A. S., Abiona, T. C., Balogun, J. A., \& Lukobo-Durrell, M. (2009). HIV sexual risk behaviors and perception of risk among college students: Implications for planning interventions. BMC Public Health, 9. doi:10.1186/1471-2458-9-281

Amaro, F., Frazão, C., Pereira, M. E., \& Teles, L. C. (2004). HIV/Aids risk perception, attitudes and sexual behaviour in Portugal. International Journal of STD \& Aids, 15, 56-60. doi:10.1258/095646204322637281

Ayala, G., Rivas, D., \& Bingham, T. (2005). Prevention heuristics and other factors that facilitate heightened HIV risk for Latino men who have sex with men in Los Angeles County. National HIV Prevention Conference, 12-15. Retrieved from http:// ww1 .aegis.org/conferences/NHIVPC/2005/T2-A1502.html
Bertoldo, R. B., \& Barbará, A. (2006). Representação social do namoro: A intimidade na visão dos jovens. Psico-USF, 11(2), 229-237. Recuperado em http://www.scielo.br/pdf/ pusf/v11n2/v11n2a11.pdf

Bogart, L. M., Kral, A. H., Scott, A., Anderson, R., Flynn, N., Gilbert, M. L., \& Bluthenthal, R. N. (2005). Condom attitudes and behaviors among injection drug users participating in California syringe exchange programs. Aids and Behavior, 9(4), 423-432. doi:10.1007/s10461-005-9014-1

Braun, V. (2013). 'Proper sex without annoying things': Anticondom discourse and the 'nature' of (hetero)sex. Sexualities, 16(3-4), 361-382. doi:10.1177/1363460713479752

Bromnick, R., \& Swinburn, P. (2003). Young people's representations of a sexual experience. Journal of Adolescence, 26, 375-379. doi:10.1016/S0140-1971(03)00017-4

Camargo, B. V., \& Bousfield, A. B. S. (2009). Social representations, risk behaviors and aids. Spanish Journal of Psychology, 12(2), 565-575. doi:10.1017/S1138741600001931

Camargo, B. V., Barbará, A., \& Bertoldo, R. B. (2007). Concepção pragmática e científica dos adolescentes sobre a Aids. Psicologia em Estudo, 12(2), 277-284. doi:10.1590/S141373722007000200008

Carvalho, M. C. R., Accioly, H., Jr., \& Raffin, F. N. (2006). Representações sociais do medicamento genérico por consumidores residentes em Natal, Rio Grande do Norte, Brasil. Cadernos de Saúde Pública, 22(3), 653-661. doi:10.1590/ S1516-93322006000400012

Castro-Vazquez, G., \& Kishi, I. (2007). Silence, condoms, and masculinity - Heterosexual Japanese males negotiating contraception. Men and Masculinities, 10, 153-177. doi: $10.1177 / 1097184 x 04264633$

Cromack, L. M. F., Bursztyn, I., \& Tura, L. F. R. (2009). The look of teenagers about health: A study on social representation. Ciência \& Saúde Coletiva, 14(2), 627-634. Retrieved from http://www.scielo.br/pdf/csc/v14n2/a31v14n2.pdf

Flament, C. (2001). Estrutura e dinâmica das representações sociais. In D. Jodelet (Ed.), As representações sociais (pp. 173-186). Rio de Janeiro, RJ: Editora da Universidade do Estado do Rio de Janeiro.

Gebhardt, W. A., Kuyper, 1., \& Greunsven, G. (2003). Need for intimacy in relationships and motives for sex as determinants of adolescent condom use. Journal of Adolescent Health, 33 , 154-164. doi:10.1016/S1054-139X(03)00137-X

Giacomozzi, A. I., \& Camargo, B. (2004). Eu confio no meu marido: Estudo da representação social de mulheres com parceiro fixo sobre prevenção de Aids. Psicologia: Teoria e Prática, 6(1), 31-44. Recuperado em http://pepsic.bvsalud. org/scielo.php?pid=S1516-36872004000100003\&script=sci arttext\&tlng=en

Giacomozzi, A. I., \& Camargo, B. (2011). Vulnerabilidade de adolescentes afrodescendentes e caucasianos em relação ao HIV/Aids. Psicologia, Saúde \& Doenças, 12, 150-158. Recuperado em http://www.scielo.gpeari.mctes.pt/pdf/psd/ v12n1/v12n1a10.pdf

Gomes, A., \& Nunes, C. (2011a). Representação social do sexo nos jornais e revistas portuguesas. Revista Interamericana de Psicologia/Interamerican Journal of Psychology, 45(1), 11-19. Recuperado em http:/www.psicorip.org/Resumos/ PerP/RIP/RIP041a5/RIP04503.pdf

Gomes, A., \& Nunes, C. (2011b). Caracterização do uso do preservativo em jovens adultos portugueses. Análise Psicológica, 29(4), 489-503. Recuperado em http://publicacoes.ispa.pt/ publicacoes/index.php/ap/article/view/99 
Gomes, A., Oliveira, D., \& Sá, C. (2008). Social representations of the Brazilian national health care system in the city of Rio de Janeiro, Brazil, according to the structural approach. Revista Latino-Americana de Enfermagem, 16(1), 122-129. doi:10.1590/S0104-11692008000100019

Goodwin, R., Kwiatkowska, A., Realo, A., Kozlova, A., Luu, L. A. N., \& Nizharadze, G. (2004). Social representations of HIV/Aids in five Central European and Eastern European countries: A multidimensional analysis. Aids Care, 16(6), 669-680. doi:10.1080/09540120412331269521

Instituto Nacional de Estatística. (2011). Censos portugueses: Principais resultados. Lisboa, Portugal: Autor. Recuperado em www.ine.pt

Instituto Nacional de Saúde. (2013). Infecção HIV/Aids - A situação em Portugal a 31 de dezembro de 2012. Lisboa, Portugal: Ministério da Saúde. Recuperado em http://repositorio.insa. pt/handle/10400.18/984

Jodelet, D. (1989). Folies et représentations sociales. Paris: Presses Universitaires de France.

Joint United Nations Program on HIV/AIDS. (2012). Report on the global aids epidemic 2012. Geneva, Switzerland: Author.

Junique, C., Barbry, W., Scano, S., Zeliger, R., \& Vergès, P. (2002). Ensemble de programmes permettant l'analysus de similitude de questionnaires et de donnes numeriques SIMI2000 (Manuel). Paris: Aix en Provence Université

Kaneko, N. (2007). Association between condom use and perceived barriers to and self-efficacy of safe sex among young women in Japan. Nursing \& Health Sciences, 9(4), 284-289. doi:10.1111/j.1442-2018.2007.00338.x

Kayiki, S. P., \& Forste, R. (2012). HIV/Aids related knowledge and perceived risk associated with condom use among adolescents in Uganda. African Journal of Reproductive Health, 15(1), 57-64. Retrieved from http://web.ebscohost.com/ehost/ pdfviewer/pdfviewer?sid=64b942ff-08ca-42a3-8ed4-6fe62 1 $92635 \mathrm{a} \% 40$ sessionmgr $15 \&$ vid $=4 \&$ hid $=24$

Manuel, S. (2005). Obstacles to condom use among secondary school students in Maputo city, Mozambique. Culture Health \& Sexuality, 7(3), 293-302. doi:10.1080/13691050 412331321302

Matson, P. A., Adler, N. E., Milsstein, S. G., Tschann, J. M., \& Ellen, J. M. (2011). Developmental changes in condom use among urban adolescents females: Influence of partner context. Journal of Adolescents Health, 48, 386-390. doi:10.1016/j.jadohealth.2010.07.016

Ministério da Saúde. (2005). HIV/Aids epidemic status at-a-glance (Portugal National Report 2005). Lisboa, Portugal: Coordenação Nacional para a Infeção de HIV/Aids, Ministério da Saúde. Retrieved from http://www.portaldasaude.pt/NR/ rdonlyres/33145068-35B6-4513-8313-1E48DD71AD35/0/ i005743_Relat\%C3\%B3rioNacional2005.pdf

Morin, M., \& Vergès, P. (1992). Enquête sur une représentation en voie d'émancipation: le Aids pour les jeunes. Les Cahiers Internationaux de Psychologie Sociale, 15, 46-75.

Moscovici, M. (1961). La psychanalyse, son image et son publique. Paris: Presses Universitaires de France.

Muñoz-Silva, A., Sánchez-García, M., Martins, A., \& Nunes, C. (2009). Gender differences in HIV-related sexual behavior among College Students from Spain and Portugal. The Spanish Journal of Psychology, 12(2), 485-495. doi:10.1017/ S1138741600001864

Oliveira, M. L., Mendes, C. M., Tardin, R. T., Cunha, M. D., \& Arruda, A. (2003). Social representation of Hansens's disease thirty years after the term leprosy was replaced in Brazil. História, Ciências, Saúde, 10(1), 41-48. doi:10.1590/ S0104-59702003000400003
Oltramari, L. C., \& Camargo, B. V. (2004). Representação social de mulheres profissionais do sexo sobre a Aids. Estudos de Psicologia (Natal), 9(2), 317-324. Recuperado em http:// www.scielo.br/pdf/epsic/v9n2/a13v9n2.pdf

Pires, M. R., \& Balieiro, C. R. (2012). Representações sobre DST/Aids e o uso de preservativo nas relações sexuais entre homens heterossexuais. Saúde e Transformação Social, 3(3), 89-104. Recuperado em http://www.incubadora.ufsc.br/index. php/saudeetransformacao/article/view/1515/2603

Reis, M., Ramiro, L., \& Matos, M. G. (2009). Contracepção, parceiros ocasionais e consumo de substâncias em jovens portugueses. Revista Lusófona de Ciências eTecnologias da Saúde, 6(2), 206-214. Recuperado em http://hdl.handle. net/10437/2235

Reis, M., Ramiro, L., Matos, M. G., \& Diniz, J. A. (2013). Determinants influencing male condom use among university students in Portugal. International Journal of Sexual Health, 25(2), 115-127. doi:10.1080/19317611.2012.728554

Ribeiro, M. L., \& Jutras, F. (2006). Representações sociais de professores sobre afetividade. Estudos de Psicologia (Campinas), 23(1), 39-45. doi:10.1590/S0103-166X2006000100005

Scano, S., Junique, C., \& Vergès, P. (2002). Ensemble de programmes permettant l'analyse des evocations - EVOC 2000 (Manuel). Paris: Aix en Provence Université

Silva, I. A. S., \& Cruz, E. A. (2008). The work of the intensive care nurse: A study on the social representations structure. Revista da Escola de Enfermagem da USP, 42(3), 546-553. Recuperado em http://www.scielo.br/pdf/reeusp/v42n3/ en_v42n3a19.pdf

Tura, L. F. R., Madeira, M. C., Silva, A. O., Gaze, R., \& Carvalho, D. M. (2008). Representações sociais de hepatites e profissionais de saúde: Contribuições para um (re)pensar da formação. Ciência, Cuidado e Saúde, 7(2), 207-215. doi:10.4025/cienccuidsaude.v7i2.5003

Vala, J. (2004). Representações sociais e Psicologia Social do conhecimento do quotidiano. In J. Vala \& M. B. Monteiro (Eds.), Psicologia Social (6. ed.). Lisboa, Portugal: Função Calouste Gulbenkian.

Vergès, P. (1992). L'evocation de l'argent: une méthode pour la définition du noyau central d'une représentation. Bulletin de Psychologie, 45(405), 203-209.

Vergès P. (1994). Approche du noyau central: propriétés quantitatives et structurales. In C. Guimelli (Ed.), Structures et transformations des représentations sociales (pp. 233-249). Lausanne, Switzerland: Delachaux et Niestlé.

Vergès, P., Tyszka, T., \& Vergès, P. (1994). Noyau central, saillance et propriétés structurales. Papers on Social Representations, 3(1), 3-12. Retrieved from http://www.psych.lse. ac.uk/psr/PSR1994/3_1994Verg1.pdf

Wachelke, J. F. R. (2009). Social Representations Centrality Index from Evocations (INCEV): An example of application on the study of the social representation on aging. Psicologia:Reflexão e Critica, 22(1), 102-110. doi:10.1590/ S0102-79722009000100014

Wachelke, J. F. R., \& Camargo, B. V. (2007). Social representations, individual representations and behavior. Interamerican Journal of Psychology, 41(3), 379-390. Retrieved from http:// pepsic.bvsalud.org/pdf/rip/v41n3/v41n3a13.pdf 